\title{
Rückläufige Krankmeldungen von Arbeitslosen - bis „Hartz IV“
}

Arbeitslose weisen einen deutlich schlechteren Gesundheitszustand als Beschäftigte in vielen Untersuchungen auf. Über das Arbeitsunfähigkeitsgeschehen bei Arbeitslosen liegen aber nur wenige Informationen vor.

\section{Alfons Hollederer}

\section{Methoden}

Anhand der GKV-Statistik werden Arbeitsunfähigkeit (AU) und Krankengeldbezug (KG) von Arbeitslosen im Vergleich zu pflichtversicherten Beschäftigten für die Jahre 2000 bis 2006 analysiert.

\section{Ergebnisse}

Das Arbeitsunfähigkeitsgeschehen ist seit 2000 durch einen starken Rückgang bei pflichtversicherten Beschäftigten bis 2006 und insbesondere bei Arbeitslosen bis 2004 gekennzeichnet. Im Jahr 2004 ereigneten sich bei Arbeitslosen nur halb so viele AU-Fälle je 100 Mitglieder wie bei pflichtversicherten Beschäftigten. Die KG-Fälle je 100 Arbeitslose verminderten sich bis fast auf den Stand von je 100 pflichtversicherten Beschäftigten.

In 2006 werden für Arbeitslosengeld-Empfänger des SGB III-Rechtskreises bei Arbeitsunfähigkeit und Krankengeld aber erheblich mehr Fälle, Leistungstage und durchschnittliche Falldauern ermittelt.

\section{Diskussion}

Die Verringerung von Arbeitsunfähigkeit und Krankengeldbezug bis 2004 ist bei Arbeitslosen offensichtlich mit Gesetzesreformen verbunden. Für den Anstieg unter Arbeitslosengeld-Empfängern in 2006 besteht weiterer Forschungsbedarf.

Dr. Alfons Hollederer, Landesinstitut für Gesundheit und Arbeit NRW, Bielefeld

\section{Einleitung}

Arbeitslose weisen im Vergleich zu Beschäftigten einen signifikant schlechteren Gesundheitszustand, eine höhere Inanspruchnahme gesundheitlicher Leistungen und ein ungünstigeres Gesundheitsverhalten auf. Das zeigen die Auswertungen der nationalen Gesundheitssurveys und repräsentativen Erhebungen wie Mikrozensus oder Sozioökonomisches Panel (Überblicke ${ }^{1},{ }^{2},{ }^{3}$ u.a.) sowie zahlreiche empirische Forschungsarbeiten zu Arbeitslosigkeit und Gesundheit (vgl. Meta-Analysen ${ }^{4}, 5$ ). In Sekundäranalysen von deutschen Krankenkassendaten ist bei Arbeitslosen ein erhöhtes Risiko für vorzeitige Mortalität, das mit Dauer der Arbeitslosigkeit zunimmt, nachgewiesen $\left({ }^{6},{ }^{7},{ }^{8}\right)$. Nach heutigem Forschungsstand bestehen durch Arbeitslosigkeit verursachte Gesundheitsbeeinträchtigungen parallel zu Selektionsprozessen am Arbeitsmarkt (1-4).

Eine Reihe von Theorien versuchte die wechselseitigen Beziehungen zwischen Arbeitslosigkeit und Gesundheit zu erklären. Zu bedeutenden theoretischen Ansätzen zählen u.a.

- eine „psychische Deprivation“ aufgrund des Wegfalls nicht nur der manifesten, sondern auch latenten sozialen und psychischen Funktionen der Arbeit durch den Arbeitsplatzverlust $\left(\right.$ Jahoda $^{9}$ );

- eine „finanzielle Deprivation“ und Handlungsrestriktionen durch Belastungen und Verarmungsprozesse in Arbeitslosigkeit (Fryer ${ }^{10}$ );

- Stresskonzepte mit Arbeitslosigkeit als stresshafte Lebensphase, z.B. gemäß transaktionale Stresstheorie von Lazarus $\left({ }^{11}\right)$ oder Anforderungs- und Kontrollmodelle (vgl. $\left.{ }^{12}\right)$;

- mit Arbeitslosigkeit verbundene soziale Stigmatisierungskonzepte $\left({ }^{13}\right)$ und Viktimisierungsprozesse $\left({ }^{14}\right)$. 


\section{THEMA}

Die nachfolgenden Abschnitte fokussieren das Arbeitsunfähigkeitsgeschehen bei Arbeitslosen. Bisher liegen nur wenige Analysen über die Arbeitsunfähigkeitsfälle und dauern von Arbeitslosen in Deutschland vor $(6,7,8,15)$. Die Arbeitsunfähigkeitszeiten von Arbeitslosen werden nur von einzelnen Krankenkassen (BKK, GEK, TK) in den Gesundheitsreports routinemäßig ausgewiesen und die Gesundheitsberichterstattung des Bundes hat sie mehrere Jahre lang nicht publiziert. Die Arbeitsunfähigkeit (AU) hat große Bedeutung in ihren Konsequenzen sowohl für den versicherten Erkrankten und seinen Arbeitgeber bzw. die Agentur für Arbeit, den beteiligten Vertragsarzt und die Krankenkasse als auch volkswirtschaftlich durch Produktionsausfälle und Lohnersatzleistungen.

\section{Arbeitsunfähigkeit im Fall von Arbeitslosigkeit}

Der Gesetzgeber verpflichtet Arbeitslose in § 311 SGB III und § 56 SGB II, eine eingetretene Arbeitsunfähigkeit und deren voraussichtliche Dauer unverzüglich anzuzeigen. Spätestens vor Ablauf des dritten Kalendertages ist eine ärztliche Bescheinigung vorzulegen. Die Agentur für Arbeit ist berechtigt, die Vorlage der ärztlichen Bescheinigung auch früher zu verlangen (z.B. bei Eignungsfeststellung oder Trainingsmaßnahmen). Der Arbeitslosengeldanspruch verlängert sich nicht innerhalb der sechswöchigen Leistungsfortzahlung bei Arbeitsunfähigkeit.

Nach § 2 Abs. 1 der Arbeitsunfähigkeits-Richtlinien „liegt Arbeitsunfähigkeit vor, wenn der Versicherte auf Grund von Krankheit seine zuletzt vor der Arbeitsunfähigkeit ausgeübte Tätigkeit nicht mehr oder nur unter der Gefahr der Verschlimmerung der Erkrankung ausführen kann.“ Die Feststellung von Arbeitsunfähigkeit erfolgt für Arbeitnehmer berufs- bzw. tätigkeitsspezifisch. Bei Arbeitslosen ist die Arbeitsunfähigkeit aber weitergehend definiert. Erkrankt ein Arbeitsloser, ist er nur dann arbeitsunfähig, wenn er aus Gesundheitsgründen am Arbeitsmarkt die Arbeiten nicht mehr ausüben kann, für die er bei der Agentur für Arbeit zur Begründung seines Anspruchs auf Arbeitslosengeld zur Verfügung steht. Die Arbeitsunfähigkeit wird ausdrücklich nicht (mehr) an einer konkreten Erwerbstätigkeit oder beruflichen Tätigkeit gemessen, sondern nach der Vermittlungsfähigkeit in aktuell zumutbare Beschäftigung.

\section{Arbeitsunfähigkeit bei Arbeitslosigkeit vom 1.1.2004 bis}

\subsubsection{6}

Diese Regelung wurde mit der Neufassung der AU-Richtlinien durch Beschluss vom 1.12.2003 verschärft. Danach waren erkrankte Arbeitslose gemäß § 2 nur arbeitsunfähig, „wenn sie aufgrund einer Erkrankung nicht mehr in der Lage sind, leichte Tätigkeiten an mindestens 15 Wochenstunden zu verrichten." Bei Arbeitslosen entstand erst ein Krankengeldanspruch, wenn das Leistungsvermögen des Versicherten in weiten Teilen aufgehoben war. Inhaltlich folgte die Neufassung dem Schlüsselbegriff „Erwerbsfähigkeit“ der Hartz-Arbeitsmarktreformen (vgl. ${ }^{16}$ ). Nach $\S 8$
Abs. 1 SGB II ist erwerbsfähig, „wer nicht wegen Krankheit oder Behinderung auf absehbare Zeit außerstande ist, unter den üblichen Bedingungen des allgemeinen Arbeitsmarktes mindestens drei Stunden täglich erwerbstätig zu sein.“ Als „absehbar“ gilt eine Zeitspanne innerhalb von sechs Monaten. Wenn die Erwerbsfähigkeit wegen langfristiger Erkrankung entfällt, geht der Anspruch auf Arbeitslosengeld (Alg) II verloren.

\section{Arbeitsunfähigkeit bei Arbeitslosigkeit seit 23.12.2006}

Allerdings mussten die AU-Richtlinien mit Wirkung zum 23.12.2006 wieder novelliert werden. Das Bundessozialgericht sah in zwei Urteilen einzelne Kriterien, wie in der Praxis Arbeitsunfähigkeit bei Arbeitslosen zu beurteilen sei, nicht in Übereinstimmung mit der Sozialgesetzgebung. In seinem Urteil vom 7.12.2004 (Az. B1KR5/03R) hat das Bundessozialgericht festgestellt, dass vor allem das Kriterium der 15-Stunden-Grenze der verbliebenen Leistungsfähigkeit nicht in Einklang mit dem Sinn und Zweck der Regelungen über das Arbeitslosen zustehende Krankengeld einerseits und den einschlägigen leistungsrechtlichen Vorschriften der Arbeitslosenversicherung andererseits steht. Die Beurteilung setzt mit den seit 23.12.2006 geltenden AU-Richtlinien eine Befragung durch den Vertragsarzt voraus. Arbeitslose sind jetzt arbeitsunfähig, „wenn sie krankheitsbedingt nicht mehr in der Lage sind, leichte Arbeiten in einem zeitlichen Umfang zu verrichten, für den sie sich bei der Agentur für Arbeit zur Verfügung gestellt haben“ (§ 2 Abs. 3 AU-Richtlinien). Bei Arbeitslosen bezieht sich die Befragung auch auf den zeitlichen Umfang, z.B. volloder halbschichtig.

\section{Wegfall des Krankengeldanspruchs für Alg II-Empfänger seit} 1.1.2005

Ein arbeitsunfähig erkrankter Arbeitsloser erhielt bis zum 31.12.2004 zunächst das Arbeitslosengeld bis zur Dauer von sechs Wochen. Wenn die Krankheit länger anhielt, bekam er Krankengeld (KG) von der Krankenkasse in Höhe des bisherigen Arbeitslosengelds ( $\$ 47 b$ SGB V). Durch das sogenannte Verwaltungsvereinfachungsgesetz wurde der Krankengeldanspruch im SGB V von (steuerfinanzierten) Alg II-Empfängern rückwirkend zum 01.01.2005 ausgeschlossen und § 25 SGB II modifiziert. Alg II-Empfänger, die seitdem arbeitsunfähig erkranken, wird Alg II gewährt, solange Erwerbsfähigkeit und die übrigen Voraussetzungen vorliegen. Erwerbsfähige Hilfebedürftige sind gem. § 56 SGB II weiterhin verpflichtet, ihre Arbeitsunfähigkeit unverzüglich anzuzeigen und eine Arbeitsunfähigkeitsbescheinigung vorzulegen.

\section{Arbeitsunfähigkeit in der Gesundheitsberichterstattung des Bundes}

Wenn ein Arbeitsloser arbeitsunfähig erkrankt, werden die gesetzlichen Regelungen entsprechend für Arbeitneh- 
mer angewandt. Die Arbeitsunfähigkeit bei Arbeitslosen ist aber durch die Arbeitsunfähigkeitsrichtlinien weitergehend definiert und in Verbindung mit Arbeitsmarktreformen mehrmals fundamental in den Bewertungskriterien geändert worden. Sie wurden vor allem für den Zeitraum vom 1.1.2004 bis 23.12.2006 mit der Neufassung der AU-Richtlinien verschärft.

Das Entgeltfortzahlungsgesetz verpflichtet die behandelnden Ärzte der Krankenkasse unverzüglich eine Bescheinigung mit Befund und voraussichtlicher Dauer der Arbeitsunfähigkeit zu übermitteln. Für die Bundesstatistik werden die Verwaltungsdaten der gesetzlichen Krankenversicherung kassenartenübergreifend erfasst. Versicherungspflichtige Arbeitslose werden in den Monats- und Jahresdurchschnittszahlen der krankenversicherten Mitglieder nach Versicherungsverhältnis (KM1) und in einer Auflistung der Leistungsfälle und -zeiten von Arbeitsunfähigkeit und Krankengeld (Teil der KG2) ausgewiesen. Basis für die Meldungen der Arbeitsunfähigkeit bilden alle den Krankenkassen vorliegenden ärztlichen Bescheinigungen oder auf anderem Wege bekanntgewordenen AU-Fälle. Die Meldungen zur Arbeitsunfähigkeit und Krankengeldleistung erfolgen stichtagsbezogen zum jeweils 1. des Monats. Bei den Angaben in Kalendertagen werden sowohl Arbeits- als auch Sonn- und Feiertage mitgerechnet. Die Diagnosen, die die Arbeitsunfähigkeit begründen, sind nicht Bestandteil der GKV-Statistik.

Die AU- und KG-Daten wurden für die vorliegende Arbeit dem Datenangebot der Gesundheitsberichterstattung des Bundes (BMG) unter www.gbe-bund.de bis zum Berichtsjahr 2005 entnommen. Die Daten des Jahres 2006 wurden freundlicherweise vom Bundesministerium für Gesundheit vorab zur Verfügung gestellt. Die Anzahl der Arbeitsunfähigkeitsfälle je 100 Mitglieder basiert auf Berechnungen der Gesundheitsberichterstattung des Bundes nach folgender Formel:

\section{(AU-Fälle x 100): Mitglieder im Jahresdurchschnitt.}

Die AU-Tage je 100 Mitglieder und auch die KG-Fälle/ Tage je 100 Mitglieder werden analog berechnet.

Allerdings bezieht das BMG für die Jahre 2005 und 2006 bei den Indikatoren für je 100 Mitglieder die AU-/KG-Fälle und -Tage der Arbeitslosengeld-Empfänger noch auf alle Arbeitslose, also Arbeitslosengeld- plus Arbeitslosengeld II-Empfänger (vgl. www.gbe-bund.de). Diese Indikatoren wurden daher analog für das Jahr 2006 für die Grundgesamtheit der Arbeitslosengeld-Empfänger und anhand der monatlichen Mitgliedermeldungen der KM1-Statistik selbst berechnet.

Ein Vergleich der Meldedaten zwischen den Spitzenverbänden der Krankenkassen, den jeweiligen Jahren davor und danach sowie einzelnen Gesundheitsreports gibt klare Hinweise darauf, dass nicht alle Krankenkassen die Monatsmeldungen im Jahr 2005 informationstechnisch ad hoc anpassen und die AU- und KG-Daten von Arbeitslosengeld- und Alg II-Empfängern separieren konnten. Die Indikatoren, die auf Basis der KG2-Statistik berechnet werden, wurden daher aus Validitätsgründen in Abb. 1-4 für 2005 für Arbeitslose nicht dargestellt.

In die Untersuchung werden die GKV-pflichtversicherten Arbeitslosen und die GKV-pflichtversicherten Beschäftigten einbezogen. Keine Berücksichtigung finden die Versichertengruppen ohne Anspruch auf Krankengeld wie Rentner, Studenten, Praktikanten und Vorruheständler. Die freiwilligen GKV-Mitglieder werden ebenso ausgenommen, da im Jahr 2006 rund die Hälfte der freiwilligen Mitglieder der GKV keinen Anspruch auf Krankengeld (KG) nach der KM1-Statistik hatte und diese Gruppe auch nicht nach Erwerbstätigkeit untergliedert werden konnte. Nicht pflichtversicherte landwirtschaftliche Unternehmer sowie sonstige Pflichtmitglieder bleiben gleichfalls außerhalb der Betrachtung.

\section{Ergebnisse zu Arbeitsunfähigkeit und Krankengeldbezug in der GKV-Statistik}

Die Abbildung 1 zeigt die Abwärtsentwicklung der AU-Fälle je 100 Mitglieder nach Versichertenstatus. Sie erreichte einen Tiefststand sowohl bei pflichtversicherten Beschäftigten mit 109 AU-Fällen je 100 Mitglieder im Jahr 2006 als auch bei pflichtversicherten Arbeitslosen mit 56 AU-Fällen je 100 Mitglieder im Jahr 2004. Die Kluft zwischen Arbeitslosen und pflichtversicherten Beschäftigten vergrößerte sich zwischen 2000 und 2004, da die AU-Fälle je 100 Mitglieder bei Arbeitslosen stärker zurückgingen. In 2004 ereigneten sich bei Arbeitslosen im Durchschnitt dann nur halb so viele AU-Fälle je 100 Mitglieder als bei pflichtversicherten Beschäftigten.

Die AU-Fälle bei Arbeitslosen sind aber mit einer mittleren Falldauer von 21,9 Tagen sehr viel länger als bei pflichtversicherten Beschäftigten mit 13,0 Tagen in 2000. Die Unterschiede erklären sich nach Auswertungen verschiedener Krankenkassen zum Teil dadurch, dass Arbeitslose bei Kurzzeit- und Bagatellerkrankungen im geringeren Maße eine ärztliche Versorgung mit Krankschreibung beanspruchten $\left({ }^{17},{ }^{18}\right)$. Die mittlere Dauer der AU-Fälle nahm im weiteren Zeitverlauf bei Arbeitslosen zu und bei pflichtversicherten Beschäftigten leicht ab. Sie erhöhte sich bei Arbeitslosen allein vom Jahr 2003 zum Jahr 2004 von 21,7 auf 23,8 Tage während sie bei pflichtversicherten Beschäftigten in den beiden Jahren nahezu unverändert blieb.

Durch die stark rückläufigen AU-Fälle von Arbeitslosen sanken die AU-Tage je 100 Arbeitslose im Jahr 2003 unter den Äquivalenzwert von je 100 pflichtversicherten Beschäftigten (Abbildung 2). Obwohl sich die Zahl der Arbeitslosen in der gesetzlichen Krankenversicherung von jahresdurchschnittlich 3,4 Millionen im Jahr 2000 auf 4,2 Millionen im Jahr 2004 steigerte, reduzierte sich die Zahl der AU-Fälle bei Arbeitslosen insgesamt von rund 2.764.000 auf 2.368.000. ${ }^{19}$

Die Zeitreihen der Abbildungen1-4 lassen sich bei Arbeitslosen aus mehreren Gründen nicht durchgängig für 2005 und 20006 darstellen. Das vierte Gesetz für moderne Dienstleistungen am Arbeitsmarkt („Hartz IV“) veränderte 


\section{THEMA}

Abbildung 1: Arbeitsunfähigkeitsfälle je 100 Mitglieder im Jahresdurchschnitt

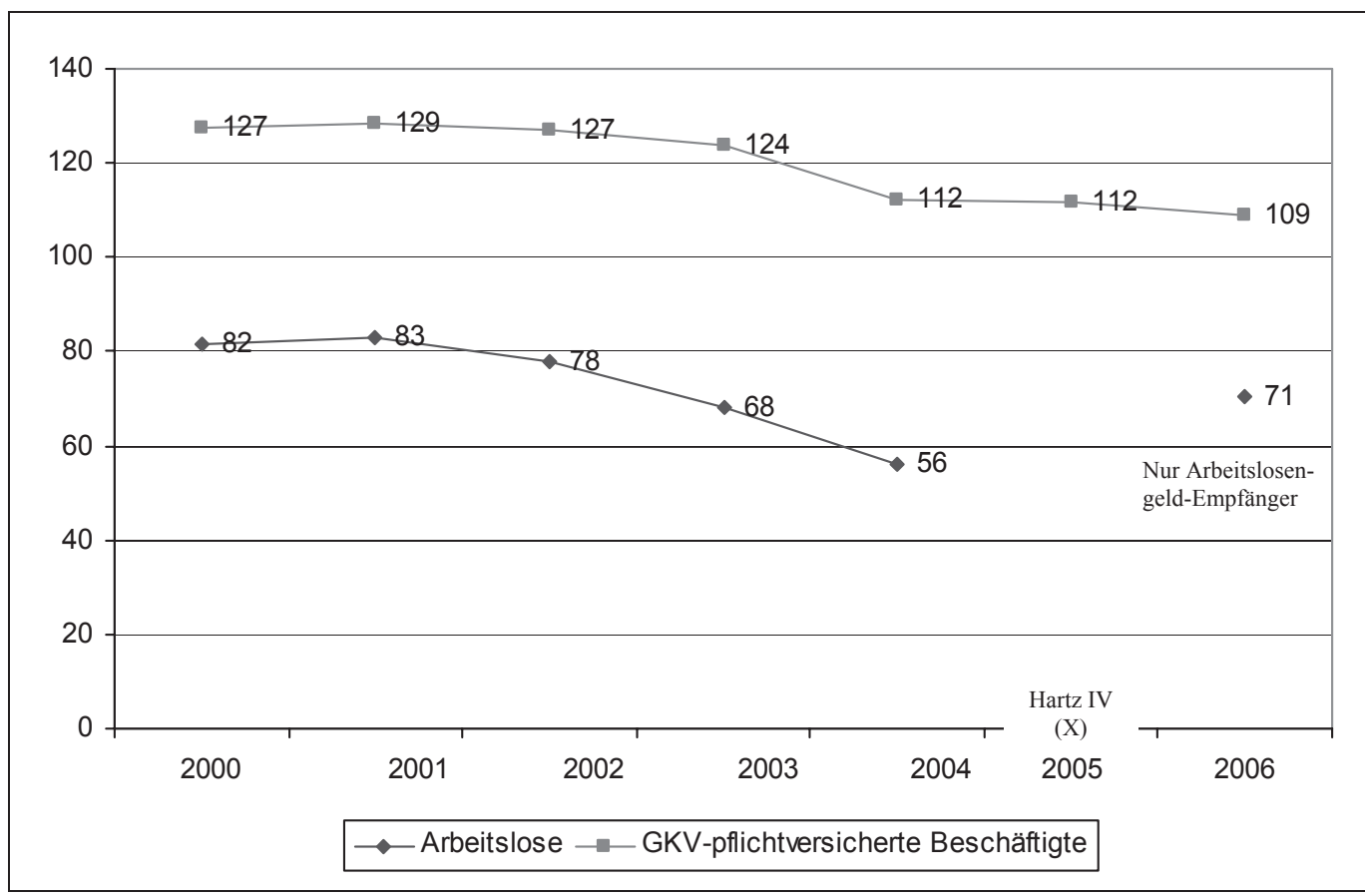

(X) Nachweis für Arbeitslose im Jahr 2005 nicht sinnvoll (siehe Methode)

Quelle: GKV-Statistik; Gesundheitsberichterstattung des Bundes; eigene Berechnung: Wert für Arbeitslosengeld-Empfänger in 2006

Abbildung 2: Arbeitsunfähigkeitstage je 100 Mitglieder im Jahresdurchschnitt

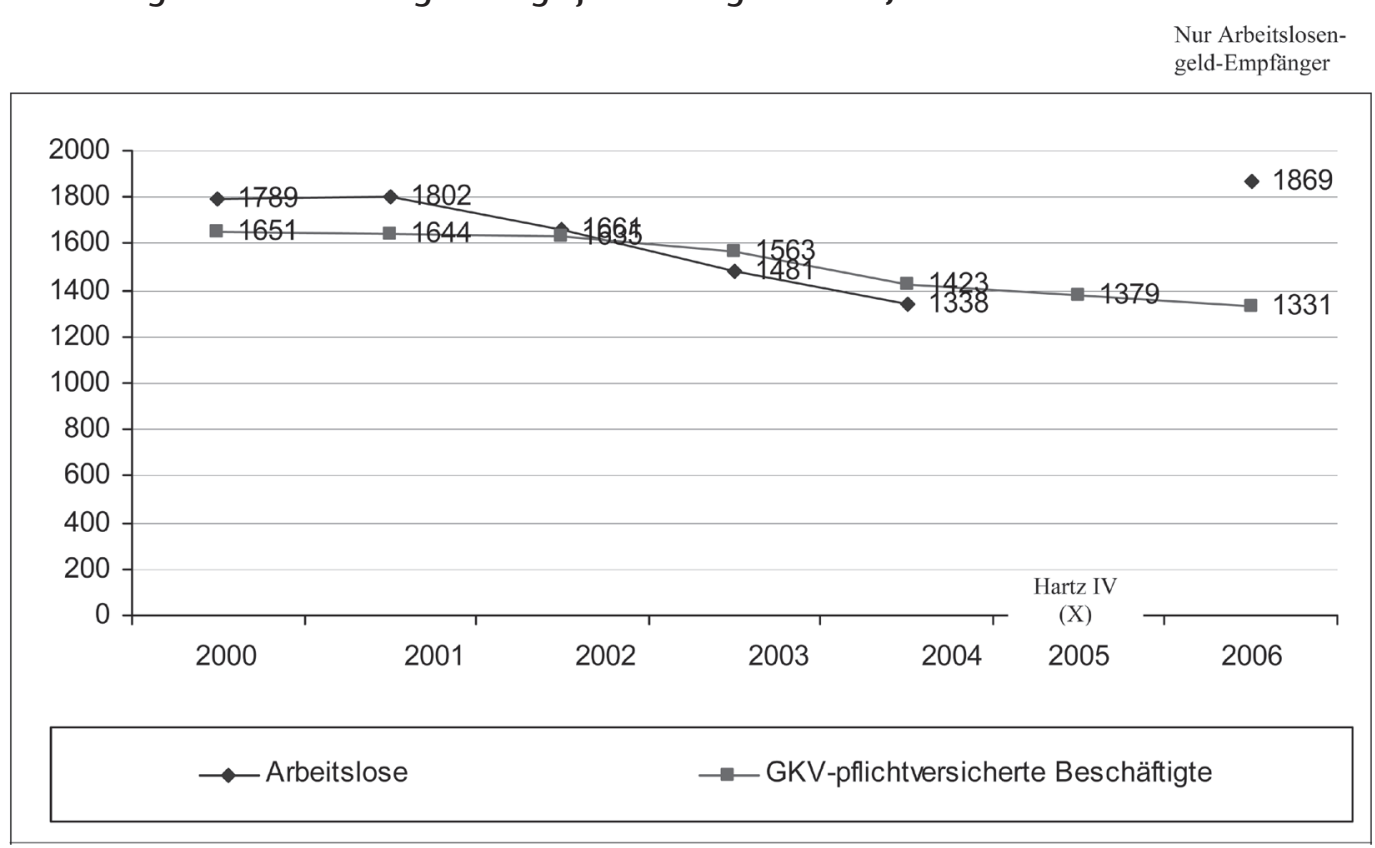

(X) Nachweis für Arbeitslose im Jahr 2005 nicht sinnvoll (siehe Methode)

Quelle: GKV-Statistik; Gesundheitsberichterstattung des Bundes; eigene Berechnung: Wert für Arbeitslosengeld-Empfänger in 2006 
in 2005 grundlegend die staatlichen Fürsorgesysteme Arbeitslosenhilfe und Sozialhilfe und vereinigte sie auf Grundlage des SGB II und SGB III mit Zu- und Abflüssen an Personengruppen. Gleichzeitig sind mit Wegfall des Krankengeldanspruchs für Alg II-Empfänger durch das so genannte „Verwaltungsvereinfachungsgesetz“ nur noch die AU- und KG-Daten der Arbeitslosengeld-Empfänger aus dem SGB III-Rechtskreis für die GKV-Statistik zu melden.

Im Jahr 2006 wurden in der GKV-Statistik jahresdurchschnittlich 4,4 Millionen Arbeitslose pflichtversichert. Darunter befanden sich 1,5 Mio. Arbeitslosengeld-Empfänger im SGB III-Rechtskreis mit rund 1.035.000 AU-Fällen. Für sie werden 2006 mehr AU-Fälle und AU-Tage je 100 Mitglieder und eine längere mittlere Falldauer (26,5 Tage) im Vergleich zum Arbeitsunfähigkeitsgeschehen bei Arbeitslosen in 2004 verzeichnet (Abbildungen 1-2).

Die Krankengeldfälle sind eine Untergruppe der AUFälle und werden in der KG2-Statistik in beiden Kategorien jeweils einmal gezählt. Sie stellen die länger andauernden und damit schwerwiegenderen Fälle dar. Der Anteil der KG-Fälle an allen AU-Fällen ist bei Arbeitslosen in etwa doppelt so hoch als analog bei den pflichtversicherten Beschäftigten.

Die KG-Fälle je 100 Arbeitslose verminderten sich bis zum Jahr 2004 auf den Stand von je 100 pflichtversicherten Beschäftigten (Abbildung 3) ${ }^{20}$. Die KG-Fälle dauerten bei Arbeitslosen mit 90,9 Tagen im Mittel aber bedeutend länger als bei pflichtversicherten Beschäftigten mit 75,7 Tagen an.

Die Differenz zwischen den KG-Tagen je 100 pflichtversicherte Beschäftigte und je 100 Arbeitslose hat sich im kurzen Zeitraum von 2000 bis 2004 mehr als halbiert (Abbildung 4).

Die Zahl der KG-Fälle je 100 Arbeitslosengeld-Empfänger erreichte im Jahr 2006 wieder die Höhe von je 100 Arbeitslosen im Jahr 2000 (Abbildung 3), allerdings mit einer höheren Zahl von KG-Tagen je 100 Mitglieder (Abbildung 4).

\section{Diskussion}

Vorab ist auf Restriktionen in der Reichweite hinzuweisen. In der GKV-Statistik gibt es eine Untererfassung von Kurzzeit-Arbeitsunfähigkeit, wenn z.B. ein Arbeitnehmer oder Arbeitsloser während der ersten drei Tage dem Arbeitgeber oder der Agentur für Arbeit keine AU-Bescheinigung vorlegen muss.

Der Stichtagsbezug der Meldungen führt zu systematischen Verzerrungen. Der jeweils erste Tag des Monats kann auch ein Samstag, Sonntag oder Feiertag mit durchschnittlich weniger AU-Fällen als an Wochentagen sein. Die Stichtagswerte ergeben auch etwas niedrigere Krankenstände im Vergleich zu Monatsdurchschnittswerten $\left({ }^{21}\right)$.

Abbildung 3: Krankengeld-Leistungsfälle je 100 Mitglieder im Jahresdurchschnitt

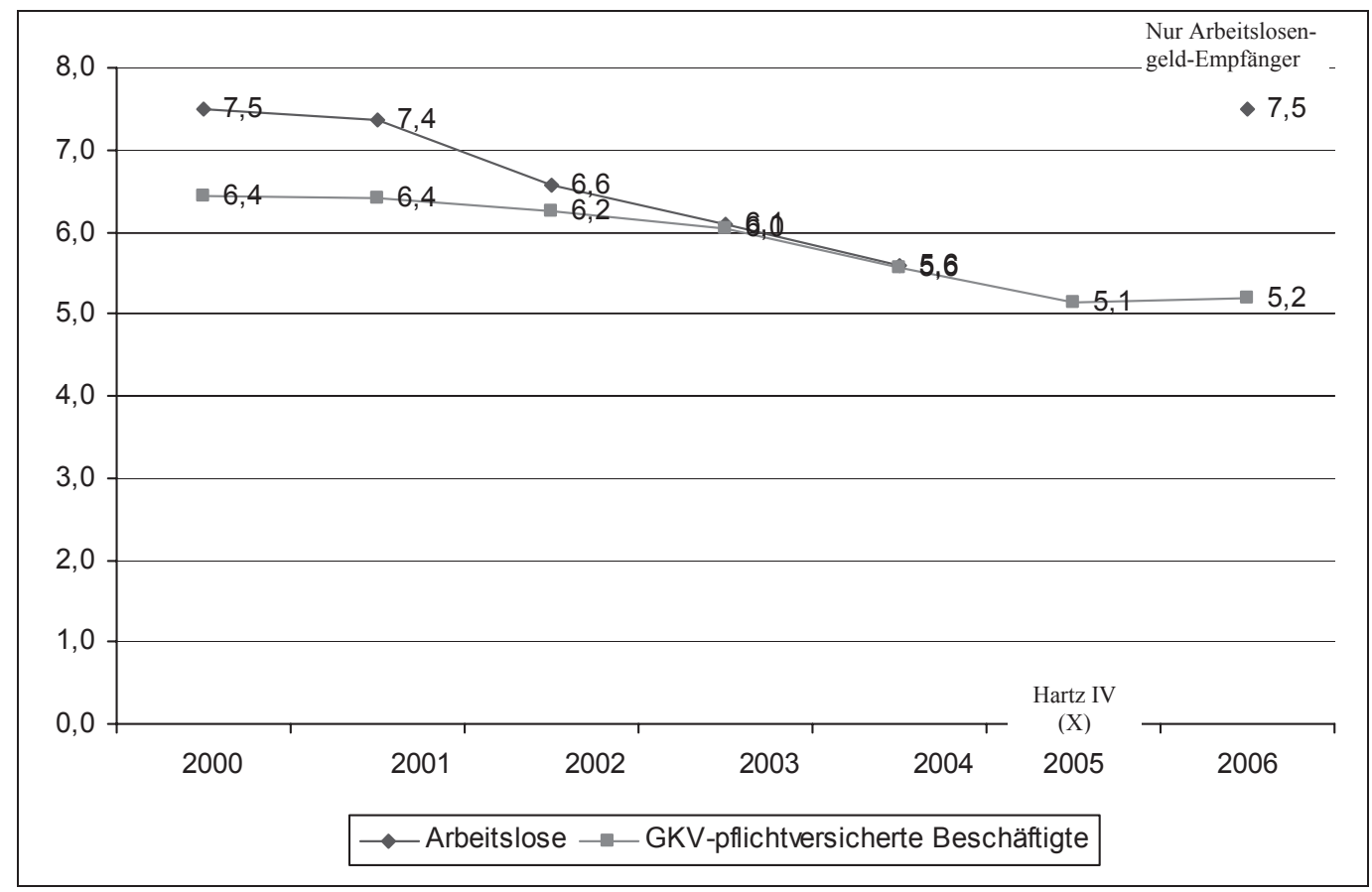

(X) Nachweis für Arbeitslose im Jahr 2005 nicht sinnvoll (siehe Methode)

Quelle: GKV-Statistik; Gesundheitsberichterstattung des Bundes; eigene Berechnung: Wert für Arbeitslosengeld-Empfänger in 2006 


\section{THEMA}

Durch die Stichtagsmeldungen entsteht eine Unschärfe bei den mittleren Falldauern, da nicht mit abgeschlossenen AU-Fällen gerechnet wird.

Die GKV-Statistik verfügt außerdem nicht über altersstandardisierte Daten. Für den Berichtszweck stellen die verwandten AU-Indikatoren aber eine gute Näherung an die Entwicklungen des Arbeitsunfähigkeitsgeschehens dar. Das gilt insbesondere für den an der Inzidenz orientierten Indikator AU-/KG-Fälle je 100 Mitglieder. Der große Vorteil der GKV-Statistik besteht in der vollständigen Erfassung der bei den Krankenkassen registrierten AU/KG-Fälle und AU/KG-Tage. Für Arbeitslose werden solche Daten ansonsten nur von sehr wenigen Krankenkassen und erst seit kurzem in der Routineberichterstattung ausgewiesen. Die Durchschnittswerte sind dabei stark von der jeweiligen Versichertenstruktur und im Zeitverlauf auch durch Fluktuation beeinflusst.

Große Sondereffekte entstanden mit der Zusammenlegung von Arbeitslosenhilfe und Sozialhilfe ab Januar 2005, die zu personellen Verschiebungen aus der Stillen Reserve in die registrierte Arbeitslosigkeit führte. Die Bundesagentur für Arbeit (BA) geht von einem zusätzlichen Arbeitskräfteangebot durch „Hartz IV“ von 380.000 Arbeitslosen im Jahresdurchschnitt 2005 aus $\left({ }^{22}\right)$. Gleichzeitig wird in der GKV-Statistik seit 2005 die Arbeitsunfähigkeit lediglich von Arbeitslosengeld-Empfängern erfasst. Die
Arbeitslosengeld-Empfänger machen nur rund ein Drittel der Arbeitslosen in 2006 aus. Damit ist ein Vergleich mit den Vorjahren nur noch sehr eingeschränkt möglich. Die GKV-Statistik hat in den früheren Jahren auch nicht nach Arbeitslosengeld- und Arbeitslosenhilfeempfängern unterschieden.

Die Entwicklung des Arbeitsunfähigkeitsgeschehens war in den letzten Jahren von einem dramatischen Rückgang des Krankenstandes in Deutschland gekennzeichnet, der bei Beschäftigten in 2006 einen historischen Tiefststand erreichte. Mit wachsender Arbeitslosigkeit steigt die Arbeitsplatzunsicherheit in den Betrieben, die Beschäftigte dazu veranlasst, Krankmeldungen tendenziell zu vermeiden $\left({ }^{23},{ }^{24}\right)$. Gleichzeitig fiel der Krankenstand von Arbeitslosen bis zum Jahr 2004 ebenfalls stark ab. Im Gegensatz zum AU-Verhalten der Beschäftigten wurde der Beitrag von Arbeitslosen bei den rückläufigen Leistungsfällen und -zeiten von Arbeitsunfähigkeit und Krankengeld bisher nicht beachtet. Die Abwärtsentwicklung im Arbeitsunfähigkeitsgeschehen ist bei Arbeitslosen aber offensichtlich zum Großteil sozialrechtlich induziert. Sie steht in Zusammenhang mit Arbeitsmarktreformen und Änderungen der AU-Richtlinien sowie parallelen Gesundheitsreformen wie der Einführung der Praxisgebühr in 2004. Darauf weist klar der singuläre Sprung der mittleren AU-Falldauern um zwei Tage bei Arbeitslosen zwischen 2003 und 2004 hin.

Abbildung 4: Krankengeld-Leistungstage je 100 Mitglieder im Jahresdurchschnitt

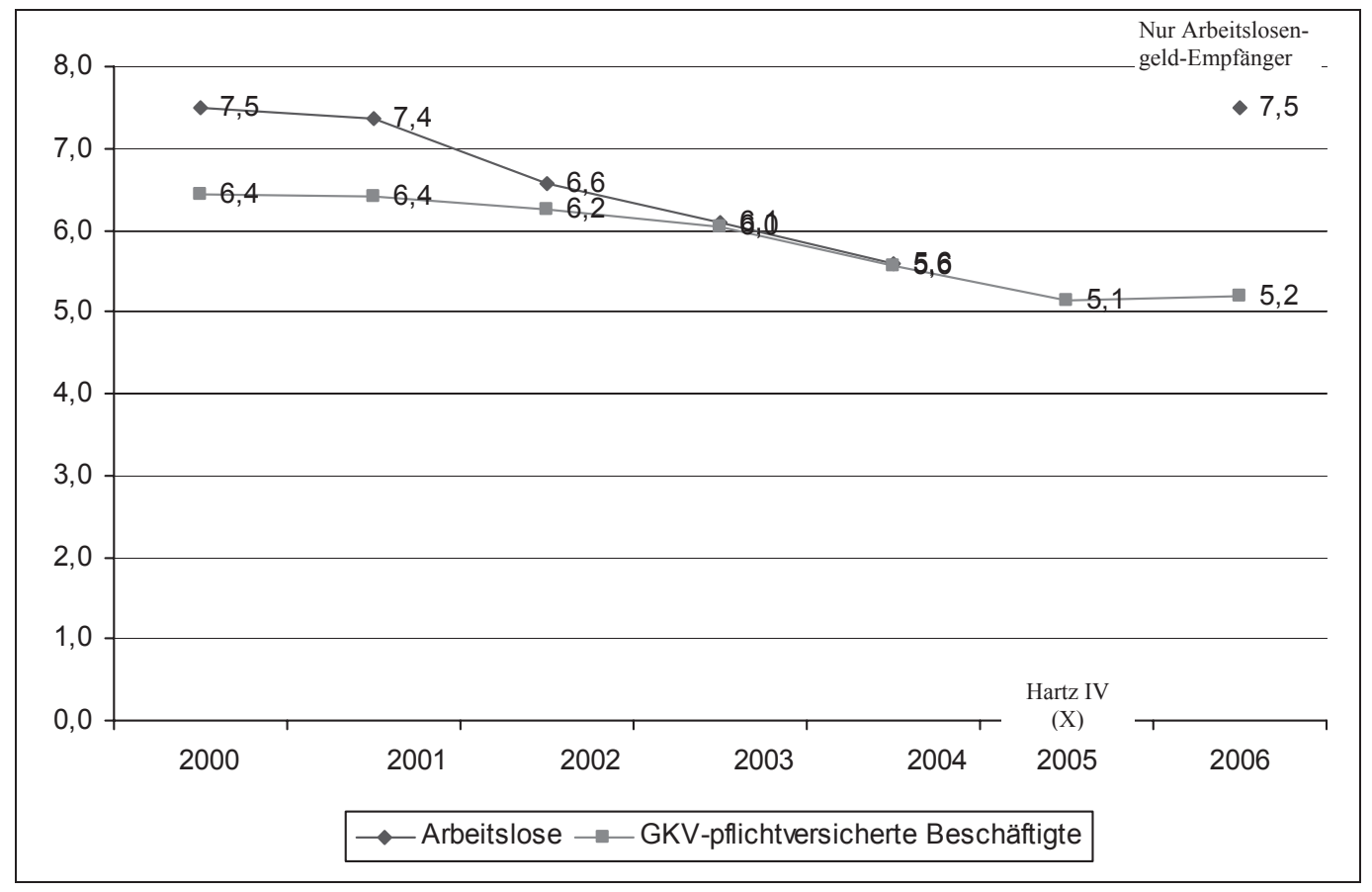

(X) Nachweis für Arbeitslose im Jahr 2005 nicht sinnvoll (siehe Methode) 
Arbeitslosengeld-Empfänger beanspruchten im Jahr 2006 die meisten AU- und KG-Tage je 100 Mitglieder im Vergleich zu allen Versichertengruppen seit 2000. Zur Erklärung des hohen Niveaus besteht weiterer Forschungsbedarf, da ihr Gesundheitszustand auf den ersten Blick sogar besser als der von Alg II-Empfängern sein müsste. Nach der Arbeitslosenstatistik lag der Anteil der gesundheitlich eingeschränkten Arbeitslosen mit 19,2\% bei den Leistungsempfängern der Arbeitslosenversicherung nach SGB III deutlich niedriger als ihr Anteil mit 23,6\% im SGB II-Rechtskreis im Jahresdurchschnitt $2006\left({ }^{20}\right)$. Außerdem waren in 2006 im SGB II-Bereich rund die Hälfte der Arbeitslosen langzeitarbeitslos, im SGB III-Bereich dagegen nur ein Viertel. Langzeitarbeitslosigkeit ist mit schlechterer Gesundheit assoziiert $\left({ }^{4}\right)$.

Auf den zweiten Blick zeigt sich, dass einer der Hauptgründe für die höhere Zahl an AU- und KG-Tage je 100 Arbeitslosengeld-Empfänger in der Altersstruktur bestehen könnte. Nach der Arbeitslosenstatistik war im SGB III-Rechtskreis ein Drittel der Arbeitslosen mindestens 50 Jahre alt, während im SBG II-Bereich dagegen nur gut ein Fünftel zu dieser Altersgruppe im Jahresdurchschnitt 2006 gehörten $\left({ }^{20}\right)$. Nach der Beschäftigtenstatistik zählten am 30.6.2006 rund 23\% der sozialversicherungspflichtig Beschäftigten mindestens 50 Jahre oder mehr. Im Jahresdurchschnitt 2004 betrug der Anteil Älterer zwischen 50 und 65 Jahren am offiziellen Arbeitslosenbestand rund ein Viertel. Wie Gesundheitsberichte der BKK dokumentieren, sind die höheren Altersgruppen durch besonders viele AUTage je 100 Versicherte charakterisiert $\left({ }^{25}\right)$.

Ein weiterer Grund könnte sein, dass der Krankengeldanspruch nicht mit dem Ende des Arbeitslosengeldbezugs wegfällt und daher die sozialrechtliche Relevanz der Arbeitsunfähigkeitsmeldung höher als im SGB II-Rechtskreis liegt.

Mit den GKV-Statistikdaten lassen sich die Ursachen für die erhöhte Arbeitsunfähigkeit und den Krankengeldbezug bei Arbeitslosengeld-Empfänger im Jahr 2006 jedenfalls nicht abschließend klären. Das bleiben zukünftigen Datenauswertungen einzelner Krankenkassen oder Forschungsarbeiten überlassen.

Der Rückgang der AU-Fälle von Arbeitslosen bis 2004 bedeutet weniger Arztkontakte. Die Arbeitsunfähigkeit ist Anlass für ein Arztgespräch, da ihre Beurteilung eine Befragung durch den Vertragsarzt voraussetzt. Bei nachlassenden Arztkontakten wächst die Gefahr einer Krankheitsverschleppung mit schwerwiegenderen Verläufen und einer Zunahme kostspieliger Krankenhausaufenthalte. Auf eine solche Entwicklung deutet der jüngste Anstieg der Krankenhausfälle von BKK-pflichtversicherten Arbeitslosen zwischen 2005 und 2006 hin $\left({ }^{23},{ }^{26}\right)$. Im Jahresdurchschnitt 2006 wurden BKK-versicherte Arbeitslose fast doppelt so oft wie BKK-pflichtversicherte Beschäftigte stationär behandelt und verbrachten mehr als zweimal so viele Tage im Krankenhaus.

Die Arbeitsunfähigkeit ist im Kontext der Gesundheitsprobleme von Arbeitslosen zu sehen, die sich als ein we- sentliches und eigenständiges Vermittlungshemmnis für die Wiedereingliederung am Arbeitsmarkt herausgestellt haben $\left({ }^{27},{ }^{28}\right)$. Gesundheitliche Einschränkungen behindern bereits die Suche nach einem neuen Arbeitsplatz $(25,26)$. Der Sachverständigenrat im Gesundheitswesen empfiehlt daher u.a. im aktuellen Gutachten $2007\left({ }^{29}\right)$ :

1. Die Verankerung des Präventionsgedankens in SGB II und SGB III.

2. Eine Aufnahme aller Erwerbslosen mit schwerwiegenden, d.h. mit vermittlungsrelevanten Gesundheitsproblemen direkt in das Fallmanagement der Arbeitsförderung.

3. Die Erweiterung und Neuorientierung der Aufgaben der Ärztlichen Dienste der BA und des Öffentlichen Gesundheitsdienstes für Klärung von Präventionsbedarf, Ausgestaltung und Koordination von Maßnahmen sekundärer und tertiärer Prävention.

4. Eine Erweiterung der Finanzierungsverantwortung für diesen Bereich - z.B. im geplanten Präventionsgesetz auch auf die BA.

Die Empfehlungen des Sachverständigenrats zielen darauf, die „Krankheiten“ und nicht allein die „Krankmeldungen" zu vermeiden.

\section{Fußnoten:}

1 Hollederer A, Brand H (Hrsg): Arbeitslosigkeit, Gesundheit und Krankheit. Bern: Huber Verlag 2006.

2 Weber A, Hörmann G, Heipertz W: Arbeitslosigkeit und Gesundheit aus sozialmedizinischer Sicht. Dtsch Arztebl 2007; 104(43): A2957-62.

3 Hollederer, A. (2008). Psychische Gesundheit im Fall von Arbeitslosigkeit. Zeitschrift Praktische Arbeitsmedizin, Ausgabe 12, Juli 2008 , S. 29-32.

4 McKee-Ryan FM, Song Z, Wanberg CR, Kinicki AJ: Psychological and physical well-being during unemployment. Journal of Applied Psychology 2005; 90: 53-76.

5 Paul KI, Hassel A, Moser K: Die Auswirkungen von Arbeitslosigkeit auf die psychische Gesundheit. In: Hollederer A, Brand H (Hrsg): Arbeitslosigkeit, Gesundheit und Krankheit. Bern: Huber Verlag 2006: 35-51.

6 Schach E, Schach S, Wille L, Glimm E, Rister-Mende S: Die Gesundheit von Arbeitslosen und Erwerbstätigen im Vergleich. Forschungsbericht Fb 708. Bremerhaven: Wirtschaftsverlag NW 1994

7 Grobe T, Schwartz F: Arbeitslosigkeit und Gesundheit. Robert KochInstitut (Hrsg). Gesundheitsberichterstattung des Bundes Heft 13. Berlin 2003.

8 Grobe T: Sterben Arbeitslose früher? In: Hollederer A, Brand H (Hrsg): Arbeitslosigkeit, Gesundheit und Krankheit. Bern: Huber Verlag 2006: 75-83.

9 Jahoda M: Wieviel Arbeit braucht der Mensch? Weinheim: Beltz Verlag 1983.

10 Fryer D: Employment deprivation and personal agency during unemployment: A critical discussion of Jahoda's Explanation of the psychological effects. Social Behavior1986; 1(3): 3-23.

11 Lazarus RS: Psychological stress and the coping process. New York: McGraw-Hill 1966.

12 Creed PA, Bartrum D: Explanations for deteriorating wellbeing in unemployed people: specific unemployment theories and beyond. In: Kieselbach T, Winefield AH, Boyd C, Anderson S (Eds): Unemployment and Health. Bowen Hills Qld: Australian Academic Press 2006: 1-20.

13 Goffman E: Stigma: Über Techniken der Bewältigung beschädigter Identität. Frankfurt: Suhrkamp Verlag 1967.

14 Kieselbach T: Outplacement als Chance. Blätter der Wohlfahrtspflege Deutsche Zeitschrift für Sozialarbeit 7+7/1998: 152-4.

15 Hollederer A: Arbeitslosigkeit und Gesundheit. IAB (Hrsg) MittAB 3/2002: 411-428. 
16 Rixen S: Erwerbsfähigkeit als Schlüsselbegriff der Arbeitsmarktreform, insbesondere im SBG II. info also 4/2006: 153-161.

17 BKK Bundesverband (Hrsg): Gesundheitsreport 2005. Essen 2005.

18 Techniker Krankenkasse (Hrsg): Gesundheitsreport 2006. Norderstedt: Merkur-Druck 2006

19 Bei pflichtversicherten Beschäftigten verringerten sich die AU-Fälle von rund 31.458.000 im Jahr 2000 auf 26.214.000 im Jahr 2004 bzw. 24.931.000 im Jahr 2006.

20 Die Zahl der KG-Fälle bei Arbeitslosen reduzierte sich von rund 254.000 im Jahr 2000 auf 235.000 im Jahr 2004. Bei den pflichtversicherten Beschäftigten verringerten sich die KG-Fälle von rund 1.587.000 im Jahr 2000 auf 1.295.000 im Jahr 2004 (bzw. 1.192.000 im Jahr 2006).

21 Priester K: Im Jahr 2006 niedrigster Krankenstand seit Einführung der Lohnfortzahlung im Krankheitsfall. Gute Arbeit 2/2007: 5-9

22 Bundesagentur für Arbeit: Arbeitsmarkt 2006. Amtliche Nachrichten der Bundesagentur für Arbeit. Nürnberg: Juli 2007.
23 Zok K: Das Arzt-Inanspruchnahmeverhalten nach Einführung der Praxisgebühr. WIdO-Monitor 2005; 2(2):1-7.

24 Zok K: Krank zur Arbeit. In: Badura B, Schröder H, Vetter C (Hrsg): Fehlzeitenreport 2007. Heidelberg: Springer 2008: 121-143.

25 BKK Bundesverband (Hrsg): Gesundheitsreport 2007. Essen 2007.

26 BKK Bundesverband (Hrsg): Gesundheitsreport 2006. Essen 2006.

27 Cramer R, Gilberg R, Hess D, Marwinski K, Schröder H, Smid M. Suchintensität und Einstellungen Arbeitsloser. IAB (Hrsg): BeitrAB 261. Nürnberg: 2002.

28 Hollederer A: Arbeitslos - Gesundheit los - chancenlos? IAB-Kurzbericht: März 2003.

29 Sachverständigenrat zur Begutachtung der Entwicklung im Gesundheitswesen (SVR) (2007). Kooperation und Verantwortung. Voraussetzungen einer zielorientierten Gesundheitsversorgung. Gutachten 2007, Berlin.

\section{Gesundheitsökonomische Beiträge}

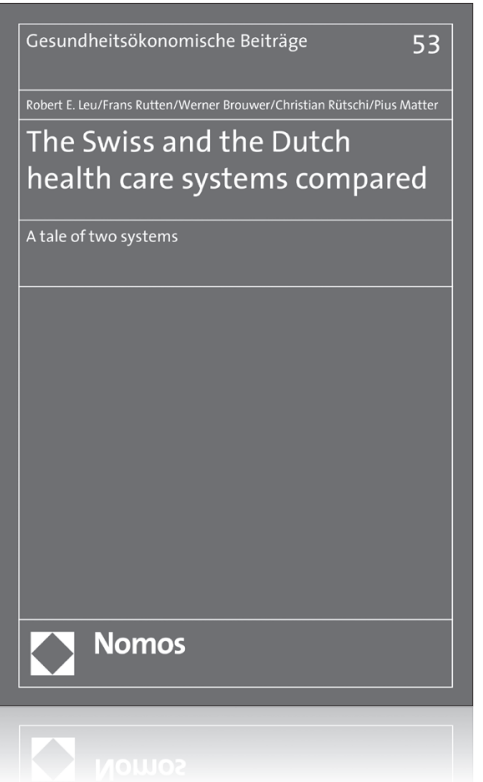

\section{The Swiss and the Dutch \\ health care systems compared}

A tale of two systems

Von Prof. Dr. Robert E. Leu, Frans Rutten, MSc, PhD, Werner Brouwer, MSc, PhD, Christian Rütschi und Pius Matter

2008, Band 53, 168 S., brosch., 29,- $€$, ISBN 978-3-8329-3739-3

In dieser Studie werden das holländische und das schweizerische Gesundheitssystem detailliert beschrieben und miteinander verglichen. Gemeinsam ist ihnen, dass sie sich im Versicherungsmarkt auf den regulierten Wettbewerb abstützen. Herausgearbeitet werden insbesondere die Elemente der beiden Systeme, die sich in der Vergangenheit bewährt haben.

Teil I der Studie gibt einen Überblick über das schweizerische und das holländische Gesundheitssystem. Teil II enthält einen detaillierteren Vergleich ausgewählter Elemente der beiden Systeme. Diskutiert werden insbesondere die Durchsetzung des Versicherungszwangs, der Leistungskatalog in der Grundversicherung, der Krankenversicherungsmarkt, Prämienunterschiede, Konsumentenmobilität, Risikoausgleich, Kostenselbstbeteiligung, Managed Care Modelle, Qualität der medizinischen Leistungen, Vertragsfreiheit, grenzüberschreitende Leistungen und Prävention. Teil III enthält eine Zusammenfassung und die wichtigsten Schlussfolgerungen. Die Langzeitpflege in der Schweiz wird im Appendix dargestellt.

Bitte bestellen Sie im Buchhandel oder

Nomos 